\title{
Estimulación cognitiva para pacientes con trastorno neurocognitivo mayor por enfermedad de Alzheimer: revisión sistemática
}

\author{
Cognitive stimulation in Alzheimer disease patients: a systematical \\ review
}

\begin{abstract}
María Camila Orejarena-Ballestas ${ }^{1}$, Ana Milena Quiñonez-Pérez ${ }^{2}$, Alejandro Marín-Gutiérrez ${ }^{3}$
${ }^{1}$ Estudiante en formación del programa de Psicología, Universidad de La Sabana. https://orcid.org/00000003-3243-4025

2Estudiante en formación del programa de Psicología, Universidad de La Sabana. https://orcid.org/00000002-6997-110X

${ }^{3}$ Doctor en Neuropsicología Clínica por la Universidad de Salamanca, España; Magíster en Neurociencia cognitiva por la Universidad de La Laguna, Colombia; Psicólogo por la Universidad Nacional de Colombia. Profesor asistente de la Universidad de La Sabana. Identificador de autor: https://www.researchgate. net/profile/Alejandro_Marin_Gutierrez; autor corresponsal: alejandro.marin.g@gmail.com; alejandro. marin2@unisabana.edu.co
\end{abstract}

Recibido: 22-11-2017; Revisado: 02-12-2017; Aceptado: 20-12-2017

\section{Resumen}

El estudio presenta los resultados de la revisión sistemática sobre la estimulación cognitiva en el trastorno cognitivo mayor por enfermedad de Alzheimer. Se desprende que ésta encabeza las intervenciones no farmacológicas de preferencia, estando dirigida a promover el mantenimiento del estado cognitivo de las personas con condiciones neurodegenerativas. Se evidencia una tendencia a comprobar la eficacia de esta terapia, teniendo efectos positivos en la calidad de vida del paciente. Sin embargo, la heterogeneidad metodológica conlleva a diversas falencias estadísticas en varios estudios, más una ausencia de evaluación de eficacia de las tareas específicas incluidas en estos programas, lo que limita la comprobación definitiva de la eficacia de estas terapias. Se discute la relevancia del estudio del campo, considerando el contexto nacional que enmarca la labor de los profesionales, y la problemática de salud pública que caracteriza esta enfermedad.

Palabras clave: estimulación cognitiva, enfermedad de Alzheimer, trastornos neurocognitivo mayor, demencia. 
Orejarena-Ballestas et al. Estimulación para pacientes con trastorno por enfermedad de Alzheimer

\section{Abstract}

This study presents the results of a systematic review concerning the cognitive stimulation in mayor cognitive disorder associated with Alzheimer disease. The main outcome shows that cognitive stimulation entails the list of non-pharmacological therapies that is addressed at to preserving the cognitive status in patients. Secondly, this review shows the lack of agreement in the literature about the efficacy of the stimulation therapy, but in most of them the benefits spreads towards an increasing of live quality. However, the lack of methodological control has lead us to think that in some cases the therapy is not efficient because the efficacy of the specific task that have been used has not been showed. we discussed the main outcomes according to our national context, where the public-health problematic is a main problematic characteristic of this kind of disease.

Keywords: cognitive stimulation, Alzheimer disease, mayor cognitive disease, dementia.

\section{Introducción}

El envejecimiento trae consigo cambios neurológicos que se expresan en dificultades sutiles para encontrar las palabras adecuadas, disminución en la velocidad de procesamiento y leves dificultades en la evocación mnésica de información, entre otras (Chodos, Johnston y Yaffe, 2014). Estos cambios no traen implicaciones en la funcionalidad de los adultos mayores, no obstante, hay un abanico de condiciones que comprometen diversas funciones cognitivas y la independencia de la persona durante el envejecimiento (Chodos, Johnston y Yaffe, 2014).

El Trastorno Neurocognitivo Mayor por Enfermedad de Alzheimer (en adelante, TNM por EA) se constituye en el DSM5 como una disminución en dos o más dominios cognitivos en comparación con el funcionamiento premórbido de la persona, siendo este referido por el consultante, un familiar o un profesional clínico, que debe evidenciarse en una evaluación neuropsicológica, y debe ser significativo para comprometer la independencia del consultante (Asociación Americana de Psiquiatría, 2014). De acuerdo con el DSM-5 el TNM por EA presenta un inicio insidioso con un curso de progresión gradual en el que el estado cognitivo de la persona se deteriora con el tiempo (Asociación Americana de Psiquiatría, 2014). Dentro de los criterios establecidos en el manual del DSM-5 de esta enfermedad, se establece que el TNM por EA puede ser probable en caso de tener evidencia de una mutación genética asociada a la aparición de esta condición o a la existencia de antecedentes familiares; o posible, cuando se cumplen los demás criterios diagnósticos, pero no hay prueba de un factor genético asociado a la sintomatología (Asociación Americana de Psiquiatría, 2014).

\section{Epidemiología}

La EA es una de las principales causas de TNM a nivel global (Huff, 2016), con una prevalencia mundial de $5 \%$ a $7 \%$ en personas mayores de sesenta años (Saykin y Rabin, 2014), siendo más frecuente en países desarrollados y afectando en mayor grado a mujeres que a hombres (Luján, 2016). Los factores de riesgo para desarrollar la EA se asocian a la edad avanzada y a los antecedentes familiares de un tipo de TNM y de EA (Kane, 2013). 
Para 2010, se estimó que alrededor de 35,6 millones de personas en todo el mundo cumplían con los criterios diagnósticos para el TNM por EA, y se estima que esta cifra se duplicará cada veinte años (Saykin y Rabin, 2014). La Asociación de Alzheimer (2017) ha decretado que 5,5 millones de estadounidenses tienen TNM por EA y que cada 66 segundos hay una persona que desarrolla esta enfermedad; cifra que se estipula cambiará para 2050, cuando cada 33 segundos se desarrollará el TNM por EA en un sujeto distinto.

Actualmente no existen estudios en Colombia sobre la prevalencia e incidencia específica del TNM por EA (Ruiz de Sánchez, Nariño y Muñoz, 2010). Sin embargo, se conoce que las cifras sobre prevalencia de TNM en este país oscilan entre el $1,8 \%$ y $3,4 \%$ en personas entre los 65 y 75 años, estableciendo que para el 2020, 6'400.000 personas en Colombia presentarán algún tipo de TNM (Ruiz de Sánchez et al., 2010).

\section{Perfil neuropsicológico del TNM por EA}

Las personas diagnosticadas con esta enfermedad presentan ciertas alteraciones en los dominios cognitivos que se asocian al sustrato neuroanatómico y molecular característico del TNM por EA (Shankar et al., 2008; Kirova, Bays y Lgalwar, 2015). Debido a que la EA es una enfermedad neurodegenerativa con un curso progresivo, se observan déficits cognitivos desde las primeras etapas. Estos serán enunciados a continuación.

En la etapa preclínica, antes de la asignación del diagnóstico de la EA, se evidencian dificultades en memoria episódica, función ejecutiva, velocidad perceptual, habilidades visoespaciales, habilidades verbales y atención (Bäckman, Jones, Berger, Jonsson y Small, 2005).
En las etapas iniciales (estadios leves), el declive en las funciones cognitivas señaladas en la etapa preclínica se hace más evidente y se acompaña de alteraciones en la independencia de actividades instrumentales de la vida diaria y dificultades significativas en otros dominios mentales (Asociación American de Psiquiatría, 2014; Kirova, Bays y Lgalwar, 2015). Se estipula que en estas fases, una persona diagnosticada con TNM por EA presenta déficits en memoria episódica debido a una dificultad para codificar y evocar información novedosa (Metzler-Baddeley y Snowden, 2005; Niu, Tan, Guan, Zhang y Wang, 2010) a partir de la degeneración neurofibrilar en la corteza entorrinal y en el hipocampo (Mesulam, 2015). A medida que esta degeneración se disemina a otras cortezas cerebrales (Mesulam, 2015), inician las alteraciones atencionales de tipo dividida y selectiva, visoespaciales (por a un componente manipulativo de memoria de trabajo), ejecutivas en la monitorización de procesos e inhibición, y lingüísticas (Kirova et al., 2015) por anomias permanentes (Romero, 2002).

Con la progresión del curso de la enfermedad, se evidencian problemas en la expresión y comprensión verbal, debido a un concretismo de pensamiento y un compromiso en el acceso al almacén léxico semántico y fonológico (Arkin, 2001), anosognosia sobre la condición de salud del paciente y apraxia del vestir, lo que compromete su independencia en actividades básicas cotidianas (Romero, 2002). Así, en las fases más avanzadas, las personas han perdido su independencia en actividades instrumentales de la vida diaria y actividades básicas de la cotidianidad, y presentan un detrimento cognitivo significativo con alteraciones en la conciencia del déficit, cambios emocionales y conductuales (Clare, Wilson, Carter, Roth y Hodges, 2004). Las funciones que se presentan 
preservadas a lo largo del curso del TNM por EA son la memoria procedimental y la cognición social (Asociación Americana de Psiquiatría, 2014).

\section{Intervención por estimulación cognitiva para TNM por EA}

Existen varios tipos de intervenciones cognitivas y conductuales para las enfermedades neurodegenerativas. Entre estas, la más popular es la estimulación cognitiva (en adelante, EC). No obstante, también se utilizan la aromaterapia con masajes (Yang, Lee, Chao, Hsu y Wang, 2016), la terapia de reminiscencia (TR), la terapia de orientación de la realidad (TOR) y la musicoterapia (Guétin et al., 2009; Woods, Aguirre, Spector y Orrell, 2012; D’Onofrio et al., 2016; Gómez y Gómez, 2017; Särkämö, 2017).

En la revisión de la literatura se enuncian distintas actividades a incluir en un programa de EC para trabajar todos los dominios cognitivos. Aunque no se encontraron resultados que indiquen qué tareas o actividades específicas se deben emplear de acuerdo con resultados estadísticos como el tamaño del efecto, a continuación se presentará las tareas expuestas en los estudios consultados.

\section{Atención}

Algunas actividades propuestas por distintos autores para la estimulación craneal (en adelante, EC) de procesos atencionales consisten en juegos de encontrar diferencias -atención sostenida y rastreo perceptual-, ordenar cartas de una baraja -atención sostenida y selectiva-, copiar ritmos auditivos -atención sostenida y selectiva(Benveniste, Jouvelot, Pin y Péquignot, 2012), copiar secuencias empleando melodías musicales -atención sostenida(Benveniste et al., 2012), realizar tareas de tachado -atención selectiva-, descifrar mensajes con códigos -atención selectiva- (Fernández-Calvo et al., 2010), emplear juegos de atención dividida con estímulos auditivos y visuales, y otros de identificación bidimensional de objetos atención sostenida y selectiva- (Aguirre et al., 2013).

\section{Memoria}

Dado que las funciones mnésicas de los pacientes con EA se observan altamente comprometidas, hay una riqueza en la diversidad de las actividades de memoria utilizadas por distintos estudios. Algunas de estas son: actividades de recuerdo y reconocimiento de imágenes (Maci, Le Pira, Quattrocchi, Di Nuovo, Perciavalle y Zappia, 2012; National Centre for Biotechnology Information, 2017); tareas de categorización -memoria semántica-; organización sintáctica de palabras para formar frases -memoria de trabajo-; inventar historias siguiendo un hilo conductor-memoria de trabajo-; entrenar estrategias y reglas mnemotécnicas como la asociación, trabajar actividades de ordenamiento de letras para formar palabras -memoria de trabajo y acceso al almacén léxico fonológico y semántico-; recordar números -memoria inmediata-; memorizar objetos contenidos en una lámina -memoria inmediata- (FernándezCalvo et al., 2010); aprendizaje de historias -entrenamiento en codificación, recuperación de memoria episódica y función ejecutiva- (Matsuda, 2007); realizar ejercicios de orientación y memoria espacial (Maci et al., 2012) y de imaginería mental; emplear técnicas de aprendizaje sin error, desvanecimiento de claves y recuperación espaciada (MetzlerBaddeley y Snowden, 2005; Olarazán et al., 2010; Matsuda, 2007; Buschert, Bokde y Hampel, 2010; Ruiz-Sánchez de León, 2012); realizar juegos de reconocimiento de canciones e intérpretes (Gómez y Gómez, 2017), y elaborar cuadernos de memoria (Loewenstein, Acevedo, Czaja y Duara, 2004). 


\section{Percepción}

Se recomienda la aplicación de actividades de exploración visual -identificación visual, atención sostenida y selectiva-; de reconocimiento facial -memoria episódica-; de discriminación visual e identificación de objetos en dos y tres dimensiones, y de identificación auditiva de estímulos, utilizando asociaciones visoverbales para trabajar estas tareas (Niu et al., 2010; Aguirre et al., 2013).

\section{Praxias}

Son pocos los estudios que especifican e incluyen tareas que estimulen el dominio de praxias directamente, conociendo que este es uno de los dominios que se ven afectados desde las primeras fases de la enfermedad. Se encontraron estudios que incluyen tareas de reconstrucción de imágenes empleando rompecabezas favoreciendo procesos construccionales y de memoria visoespacial- (Maci et al., 2012) y tareas de esquema corporal, en las que se deben imitar posturas vistas en una pantalla -praxias ideomotoras(Requena, Maestú, Campo, Fernández, Gil y Ortiz, 2007).

\section{Lenguaje}

Las alteraciones lingüísticas pueden presentarse en estadios más avanzados del TNM por EA (Arkin, 2001). No obstante, es relevante realizar una EC en este dominio desde fases primarias para contribuir con la preservación de la función y como mecanismo compensatorio para proyectar la capacidad de los circuitos alterados y su sobreactivación sináptica (Arkin, 2001). Para estimular los procesos subyacentes a este dominio, se pueden realizar actividades de presentación rápida de palabras -expresión, fluidez, acceso al almacén léxico, velocidad de procesamiento-; de discriminación fonológica, de comprensión verbal (Maci et al., 2012); de denominación escrita; de descripción espontánea de imágenes -fluidez y denominación-; de asociación de palabras con propias vivencias y recuerdos -acceso a información remota y activación de información relacionada, expresión y comprensión verbal-; de interpretación y completamiento de refranes -comprensión, memoria episódica y semántica-; juegos de denominación por demanda y cambios de series (switching set) -denominación, fluidez y flexibilidad mental-; juegos de fluidez, y bingo visual y musical, como tarea de seguimiento de otra actividad -comprensión verbal, designación, asociación y acceso al almacén léxico(Arkin, 2001; Spector et al., 2003; Matsuda, 2007; Niu et al., 2010; Gómez y Gómez, 2017).

\section{Función ejecutiva}

Para estimular los procesos ejecutivos que regulan el comportamiento dirigido a objetivos en contextos con distintos grados de complejidad social, y realizar la monitorización afectiva y cognitiva de una persona (Hart, 2010), se sugiere incluir las siguientes actividades dentro del programa de intervención: solitario -organización, planeación, solución de problemas y flexibilidad mental-, juegos de si/no con cartas u otros materiales -control inhibitorio y flexibilidad cognitiva-, tareas de secuenciación memoria de trabajo, cálculo, alternancia en flujo de información, organización y jerarquización-, actividades de identificación de causas -cognición social, anticipación y organización- (FernándezCalvo etal., 2010), actividades de solución de problemas cotidianos -solución de problemas y memoria de trabajo-, actividades dirigidas a reconocer el valor del dinero y su uso (Maci et al., 2012), y de generación de historias al observar fotografías -abstracción, flexibilidad de pensamiento y memoria de trabajo(Requena, et al., 2007; Niu et al., 2010). 
Justificación

EITNM por EA constituye una problemática de salud pública internacional debido a las consecuencias que trae para la persona diagnosticada, los requerimientos de salud y atención psicosocial, y la rehabilitación requerida para realizar una adaptación tanto de la familia como del paciente en condición de discapacidad (Cicerone et al., 2000; Shankar, 2008; Hart, 2010). El papel del profesional en neuropsicología frente a esta enfermedad es crucial, pues su acción contribuye, no solo a la determinación de la presencia de la enfermedad, sino también al establecimiento de la severidad y el curso de ésta $y$, por lo tanto, también a establecer el impacto en la funcionalidad del paciente y su nivel de independencia (Saykin y Rabin, 2016).

Por esta razón, conocer el estado del arte sobre el tema y revisar las investigaciones sistemáticamente los trabajos actuales que han mostrado eficacia en el tratamiento del paciente con TNM por EA se convierte en el objetivo principal de este trabajo.

Las razones que nos llevan a realizar este trabajo se centran en que este tipo de intervención ayuda a aplazar la institucionalización del paciente diagnosticado con esta condición, aumenta el nivel de calidad de vida de los pacientes y sus familias, y logra desacelerar el proceso de degeneración cognitiva a un bajo costo, gracias al esfuerzo humano que requiere de baja inversión en tecnología médica de alto valor (Olazarán et al., 2010; 2014; Capotosto et al., 2017).

Por otro lado, actualmente los servicios de neuropsicología en Colombia tienen la necesidad de realizar guías de estimulación cognitiva para trabajar en la intervención de pacientes diagnosticados con TNM por EA que estén basados en la evidencia. Consideramos que este trabajo podría ser relevante para motivar la investigación sobre este tema en nuestro país con una revisión actualizada y minuciosa.

\section{Metodología}

\section{Selección de los estudios}

La búsqueda de la información relevante para nuestro objetivo comenzó con la selección de las bases de datos ScienceDirect y PubMed. Las palabras clave utilizadas para consultar los distintos documentos incluidos fueron Alzheimer's Disease, Dementia, Cognitive Stimulation Therapy y Major Neurocognitive Disorder. Al realizar esta búsqueda se obtuvieron 65.511 resultados en ScienceDirect y 260 resultados en PubMed.

\section{Criterios de selección}

Se aceptaron los estudios realizados a partir del año 2001 en adelante, en inglés o español, cuyo objetivo principal fuera la eficacia de la EC en pacientes con EA o con TNM. Se incluyeron estudios de corte cuantitativo únicamente con diseños experimentales, cuasiexperimentales, y ensayos controlados aleatorizados. A partir de la selección de estos, se redujeron las búsquedas en las bases de datos a treinta artículos obtenidos en ScienceDirect y once de PubMed, para un total de 31 estudios. Adicional a esta muestra, se buscaron referencias que permitieran construir un marco teórico sobre los aspectos fisiopatológicos de la EA, los criterios diagnósticos del TNM por EA, la epidemiología de la enfermedad, el perfil cognitivo asociado a esta y una presentación sobre la intervención neuropsicológica a partir de la EC y otros procedimientos frecuentemente vistos en la literatura. Finalmente, aunque las palabras clave estuvieron en inglés, se incluyeron algunos estudios en castellano, ya que estas publicaciones también contaron con palabras clave y 
resumen en inglés. Consideramos que este paso no le quita calidad al trabajo. Por el contrario, ofrece una perspectiva del tema en contextos de habla hispana que luego pueden ser útiles a la hora de realizar futuros estudios sobre el tema.

\section{Resultados}

La tabla 1 muestra los resultados de la revisión que se realizó con los artículos seleccionados. Se presenta la tabla que integra los resultados de esta revisión con las descripciones de cada uno de los artículos consultados.

Tabla 1. Resultados de la revisión narrativa realizada

\begin{tabular}{|c|c|c|c|c|c|}
\hline Autores & Año & Método & Terapia & Detalles de la terapia & Hallazgos principales \\
\hline $\begin{array}{l}\text { Stewart, D. B., } \\
\text { Berg-Weger, } \\
\text { M., Tebb, S., } \\
\text { Sakamoto, } \\
\text { M., Roselle, } \\
\text { K., Downing, } \\
\text { L., Lundy, J. \& } \\
\text { Hayden, D. }\end{array}$ & 2017 & $\begin{array}{c}\text { Estudio } \\
\text { descriptivo } \\
\text { comparativo }\end{array}$ & $\begin{array}{c}\text { EC y Ejercicio } \\
\text { Físico }\end{array}$ & 2 semestres & $\begin{array}{l}\text { Las intervenciones multimodales pueden } \\
\text { mantener o mejorar temporalmente el } \\
\text { funcionamiento cognitivo, lingüístico, } \\
\text { social y físico de los pacientes con } \\
\text { Alzheimer. }\end{array}$ \\
\hline $\begin{array}{l}\text { Orrell, M., Yates, } \\
\text { L., Leung, P., } \\
\text { Kang, S., Hoare, } \\
\text { Z., et. al }\end{array}$ & 2017 & $\begin{array}{l}\text { Ensayo } \\
\text { controlado } \\
\text { aleatorio }\end{array}$ & $\begin{array}{l}\text { EC, TOR y } \\
\text { TR. También } \\
\text { estimulación } \\
\text { multisensorial. }\end{array}$ & $\begin{array}{l}14 \text { sesiones grupales de } \\
45 \text { minutos, dos veces por } \\
\text { semana por } 7 \text { semanas. } \\
\text { Actividades con dinero, } \\
\text { juegos de palabras, } \\
\text { personajes famosos y } \\
\text { noticias recientes. }\end{array}$ & $\begin{array}{l}\text { Grupo experimental demuestra aumento } \\
\text { en puntuaciones del ADAS-Cog y QoL- } \\
\text { AD en comparación con medida pre- } \\
\text { test y grupo control. Los resultados } \\
\text { se comparan con los de ensayos de } \\
\text { tratamiento farmacológico para TNM. }\end{array}$ \\
\hline $\begin{array}{l}\text { Capotosto, } \\
\text { E., Belacchi, } \\
\text { C., Gardini, } \\
\text { S., Faggian, } \\
\text { S., Piras, F., } \\
\text { Mantoan, V., } \\
\text { Salvalaio, E., } \\
\text { Pradelli, S. \& } \\
\text { Borella, E. }\end{array}$ & 2017 & $\begin{array}{l}\text { Ensayo } \\
\text { controlado } \\
\text { aleatorio }\end{array}$ & $\begin{array}{l}\text { Rehabilitación } \\
\text { cognitiva }\end{array}$ & $\begin{array}{l}24 \text { sesiones de } 45 \\
\text { minutos } 2 \text { veces a la } \\
\text { semana }\end{array}$ & $\begin{array}{l}\text { Demuestran un mejor desempeño } \\
\text { en tareas que fueron similares a las } \\
\text { utilizadas en el entrenamiento. Recuerdo } \\
\text { en asociaciones de nombres de rostro, } \\
\text { orientación, velocidad de procesamiento } \\
\text { cognitivo y las tareas funcionales } \\
\text { específicas estuvieron presentes } \\
\text { después de la intervención y en un } \\
\text { seguimiento de } 3 \text { meses. }\end{array}$ \\
\hline $\begin{array}{l}\text { Gómez. M. \& } \\
\text { Gómez, J. }\end{array}$ & 2017 & $\begin{array}{c}\text { Ensayo } \\
\text { controlado }\end{array}$ & $\begin{array}{c}\text { Aprendizaje } \\
\text { sin Error, } \\
\text { Aprendizaje con } \\
\text { Error }\end{array}$ & 8 días consecutivos & $\begin{array}{l}\text { EL puede ser más beneficioso para } \\
\text { los pacientes con amnesia profunda, } \\
\text { pero que las capacidades residuales de } \\
\text { memoria explícita pueden anular los } \\
\text { beneficios de EL. }\end{array}$ \\
\hline $\begin{array}{l}\text { National } \\
\text { Centre for } \\
\text { Biotechnology } \\
\text { Information }\end{array}$ & 2017 & $\begin{array}{l}\text { Ensayo } \\
\text { controlado } \\
\text { aleatorio }\end{array}$ & EC & $\begin{array}{l}20 \text { minutos de sesión } \\
\text { por } 3 \text { semanas y } 8 \text { hras } \\
\text { diarias de psicoeducación }\end{array}$ & $\begin{array}{l}\text { Tanto la terapia de estimulación cognitiva } \\
\text { clásica como la basada en computadora } \\
\text { la terapia mejora la cognición en sujetos } \\
\text { tratados con una dosis estable de ChEI, } \\
\text { en comparación a aquellos que fueron } \\
\text { tratados solo con ChEIs. }\end{array}$ \\
\hline $\begin{array}{l}\text { Yang, Y. P., Lee, } \\
\text { F. P., Chao, H. } \\
\text { C., Hsu, F. Y. \& } \\
\text { Wang, J. J. }\end{array}$ & 2016 & $\begin{array}{l}\text { Estudio de } \\
\text { cohorte con } \\
\text { medidas pre } \\
\text { y post test }\end{array}$ & $\begin{array}{c}\text { EC y } \\
\text { tratamiento con } \\
\text { donepezilo }\end{array}$ & $\begin{array}{l}\text { Sesiones individuales con } \\
\text { complejidad ajustada por } \\
\text { resultados de evaluación } \\
\text { pre-test. Interacción } \\
\text { en conversaciones, } \\
\text { psicoeducación de la EA, } \\
\text { aprendizaje de historias y } \\
\text { tareas de fluidez verbal. }\end{array}$ & $\begin{array}{l}\text { Hay cambios significativos en las pun- } \\
\text { tuaciones del Mini-mental State Exami- } \\
\text { nation en el grupo control (donepezilo), } \\
\text { pero no en el experimental (donepezi- } \\
\text { lo+EC). El grupo control presenta un } \\
\text { declive promedio mayor al experimen- } \\
\text { tal, con tamaños de efecto negativos. } \\
\text { La combinación de EC con donepezilo, } \\
\text { disminuye progresión de la EA. }\end{array}$ \\
\hline
\end{tabular}




\begin{tabular}{|c|c|c|c|c|c|}
\hline Autores & Año & Método & Terapia & Detalles de la terapia & Hallazgos principales \\
\hline $\begin{array}{l}\text { D'Onofrio, } \\
\text { G., Sancarlo, } \\
\text { D.,Seripa, D., } \\
\text { Ricciardi, F., } \\
\text { Giuliani, F., } \\
\text { Panza, F. \& } \\
\text { Greco, A. }\end{array}$ & 2016 & $\begin{array}{l}\text { Ensayo } \\
\text { controlado } \\
\text { aleatorio }\end{array}$ & $\begin{array}{c}\text { EC y } \\
\text { tratamiento con } \\
\text { donepezilo }\end{array}$ & $\begin{array}{l}\text { Sesiones grupales de } 45 \\
\text { minutos, cinco días a la } \\
\text { semana durante } 2 \text { años. } \\
\text { Se trabajaban actividades } \\
\text { de orientación, esquema } \\
\text { corporal, familia y socie- } \\
\text { dad, cuidado de sí mismo, } \\
\text { reminiscencia, actividades } \\
\text { de la vida diaria, y anima- } \\
\text { les, personas o cosas. }\end{array}$ & $\begin{array}{l}\text { Una intervención combinada entre fármaco } \\
\text { y EC logra mejorar significativamente el } \\
\text { funcionamiento del paciente con EA luego } \\
\text { del primer año, mostrando disminución } \\
\text { no significativa del deterioro cognitivo } \\
\text { luego del segundo año de tratamiento } \\
\text { combinado, sin traspasar los niveles } \\
\text { estipulados de la línea base. El grupo } \\
\text { control mostró deterioro significativo al } \\
\text { cabo de dos años. }\end{array}$ \\
\hline $\begin{array}{l}\text { D'Amico, F., } \\
\text { Rehill, A., Knapp, } \\
\text { M., Aguirre, E., } \\
\text { Donovan, H., } \\
\text { Hoare, Z., et. tal }\end{array}$ & 2015 & $\begin{array}{l}\text { Ensayo } \\
\text { controlado } \\
\text { aleatorio }\end{array}$ & EC y TOR & $\begin{array}{l}14 \text { sesiones grupales, dos } \\
\text { veces por semana por } 7 \\
\text { semanas. }\end{array}$ & $\begin{array}{l}\text { La EC tiene beneficios en cognición y } \\
\text { calidad de vida comparables con los } \\
\text { resultados de la medicación. También } \\
\text { demostró ser rentable, en comparación } \\
\text { con otros tratamientos. }\end{array}$ \\
\hline $\begin{array}{l}\text { Cove, J., Jacobi, } \\
\text { N., Donovan, H., } \\
\text { Orrell, M., Stott, } \\
\text { J. \& Spector, A. }\end{array}$ & 2014 & $\begin{array}{l}\text { Ensayo } \\
\text { controlado } \\
\text { aleatorio }\end{array}$ & EC & $\begin{array}{l}\text { entrenados en un } \\
\text { videojuego de estrategia } \\
\text { en tiempo real por } 23.5 \\
\text { horas }\end{array}$ & $\begin{array}{l}\text { Mejoraron significativamente las tareas } \\
\text { cognitivas, como la memoria de trabajo, } \\
\text { la memoria visual a corto plazo, la } \\
\text { rotación mental y funciones ejecutivas. } \\
\text { Las tendencias en la mejora también } \\
\text { se observaron, en los participantes de } \\
\text { videojuegos, en inhibición y razonamiento. }\end{array}$ \\
\hline
\end{tabular}

Orrell, M.,

Aguirre, E., Spector, A., Hoare, Z., Woods, R. T., Streater, A., Donovan, $\mathrm{H}_{\text {., }}$ Hoe, J., Knapp, M., Whitaker, C. \& Russell, I.

Yamanaka, K., Kawano, Y., Noguchi, D., Nakaaki, S., Watanabe, N., Amano, T. \& Spector, A.

Ensayo controlado aleatorio

Ensayo controlado aleatorio

EC viduales de 45 minutos a la semana durante 10

Diseño cuasiexperimental

Hall, L., Orrell, M., Stott, J. \& Spector, A. de un grupo con medida pres-test y post-test
EC,

Entrenamiento cognitivo,

rehabilitación cognitiva semanas. Se emplean tareas de pruebas neuropsicológicas (orientación, fluidez verbal, figuras superpuestas y creación de historias usando fotografías)

Dos sesiones semanales de 45 minutos cada una por 7 semanas, y una fase de mantenimiento de 16 semanas usando intervenciones basadas en la evidencia. Usan tableros de orientación a la realidad, y actividades (juegos, canciones, tareas de actualización) cuyo título exponen, pero no explican.

Se comprueba eficacia de la EC para mejorar síntomas neuropsiquiátricos de apatía y depresión, y mejores puntuaciones en el Mini-mental State Examination en pacientes con EA Leve.

El grupo de intervención de mantenimiento obtiene mejoría significativa en el funcionamiento cognitivo asociado con mejores puntuaciones en el Mini-mental State Examination. Se requieren nuevos ensayos controlados aleatorios que evalúen a gran escala los beneficios de continuar programas de EC luego de 7 semanas.

Las intervenciones cognitivas mejoran el funcionamiento cognitivo de las personas con EA, su calidad de vida, disminuyen las perturbaciones conductuales y las Intervenciones de todo habilidades en actividades cotidianas. tipo. Las duraciones La intervención cognitiva global (EC) $\begin{array}{lllll}\text { varían entre } 3 & \text { y } & 24 & \text { trae más beneficios al paciente con EA } \\ \text { meses. No especifican que otras modalidades de intervención }\end{array}$ actividades.

psicosocial. Es necesario desarrollar pautas de intervenciones cognitivas que beneficien a los pacientes con EA, considerando la futura prevalencia que tendrá la enfermedad. 
Revista Búsqueda - Julio / Diciembre de 2017

\begin{tabular}{|c|c|c|c|c|c|}
\hline Autores & Año & Método & Terapia & Detalles de la terapia & Hallazgos principales \\
\hline $\begin{array}{l}\text { Aguirre, E., } \\
\text { Hoare, Z., } \\
\text { Streater, A., } \\
\text { Spector, A., } \\
\text { Woods, B., Hoe, } \\
\text { J. \& Orrell, M. }\end{array}$ & 2013 & $\begin{array}{l}\text { Ensayo } \\
\text { controlado } \\
\text { aleatorio }\end{array}$ & $\begin{array}{l}\text { EC y } \\
\text { tratamiento con } \\
\text { donepezilo }\end{array}$ & $\begin{array}{l}\text { Sesiones de EC de } 35 \\
\text { minutos durante } 7 \\
\text { semanas }\end{array}$ & $\begin{array}{l}\text { El grupo experimental (donepezilo y } \\
\text { EC) mejoró significativamente en las } \\
\text { puntuaciones del HDS-R mientras que } \\
\text { el grupo control (donepezilo) no lo hizo. }\end{array}$ \\
\hline $\begin{array}{l}\text { Maci, T., Le Pira, } \\
\text { F., Quattrocchi, } \\
\text { G., Di Nuovo, S., } \\
\text { Perciavalle, V. \& } \\
\text { Zappia, M. }\end{array}$ & 2012 & Estudio piloto & EC & $\begin{array}{l}14 \text { sesiones durante } \\
7 \quad \text { semanas } \text { (dos } \\
\text { semanales), siguiendo } \\
\text { lineamiento de Spector et } \\
\text { al. (2005). }\end{array}$ & $\begin{array}{l}\text { Los procesos de lenguaje evaluados en } \\
\text { el ADAS-Cog mejoran significativamente } \\
\text { luego de una terapia por EC. Puede } \\
\text { explicarse por la estimulación de } \\
\text { procesos de conceptualización y la } \\
\text { generación de estrategias de asociación. }\end{array}$ \\
\hline $\begin{array}{l}\text { Woods, B., } \\
\text { Aguirre, E., } \\
\text { Spector, A. E. \& } \\
\text { Orrell, M. }\end{array}$ & 2012 & $\begin{array}{l}\text { Ensayo } \\
\text { controlado } \\
\text { aleatorio }\end{array}$ & EC & 36 sesiones de una hora & $\begin{array}{l}\text { Logró disminuir el declive cognitivo } \\
\text { y mejorar el ajuste, psicológico y } \\
\text { conductual, de los pacientes con } \\
\text { EA. Los pacientes que recibieron la } \\
\text { EC mostraron beneficios cognitivos, } \\
\text { aunque el deterioro funcional de los } \\
\text { pacientes permaneció intacto. }\end{array}$ \\
\hline
\end{tabular}

Benveniste, S., Jouvelot, P., Pin, B. \& Péquignot, $\mathrm{R}$

Niu, Y., Tan, J., Guan, J., Zhang, Z.\& Wang, L.

Aguirre, E., Spector, A., Hoe, J., Russell, I.T., Knapp, M., Woods, R.T. \& Orrell, $M$.

Buschert, V., Bokde, A. L. W. 2010 \& Hampel, $\mathrm{H}$.

\begin{tabular}{|c|c|c|}
\hline 2012 & $\begin{array}{l}\text { Ensayo } \\
\text { controlado }\end{array}$ & $\begin{array}{c}\text { EC y Ejercicio } \\
\text { físico }\end{array}$ \\
\hline
\end{tabular}
rante 3 meses. Se realiza 1 hora de ejercicio físico, 1 hora de tareas de EC Y 30 minutos de discusiones grupales. Actividades especificadas de orientación, memoria, lenguaje y función ejecutiva.

Ensayo controlado aleatorio

Ensayo

2010 controlado aleatorio

Musicotrapia

1 vez a la semana por 4 meses

Estudio de revisión narrativa
El funcionamiento cognitivo se mantiene luego de tres meses de intervención combinada, con mejoras en apatía, calidad de vida y estado de ánimo. Estos dos últimos se hacen evidentes tanto en pacientes como en cuidadores.

Se evidencia un beneficio claro y consistente sobre la función cognitiva asociada con la EC. De uno a tres meses después del final del tratamiento, se presente beneficio también. En los análisis secundarios se observaron beneficios en la calidad de vida y el bienestar informados.

Se ha demostrado que la musicoterapia es un enfoque eficaz para tratar a pacientes con demencia. Una gran mayoría de los pacientes, con demencia de leve a moderada, comprendieron y usaron la interfaz. Fueron capaces de evocar información del MINWii incluso sin un entrenamiento musical previo.

Luego de una intervención por EC se observa una mejoría en la cognición, el estado de ánimo y calidad de vida en pacientes con demencia. Se requieren ensayos controlados aleatorios con muestras más amplias. 


\begin{tabular}{|c|c|c|c|c|c|}
\hline Autores & Año & Método & Terapia & Detalles de la terapia & Hallazgos principales \\
\hline $\begin{array}{l}\text { Matsuda, O., } \\
\text { Shido, E., } \\
\text { Hashikai, A., } \\
\text { Shibuya, H., } \\
\text { Kuono, M., Hara, } \\
\text { C. \& Saito, M. }\end{array}$ & 2010 & $\begin{array}{l}\text { Ensayo } \\
\text { controlado no } \\
\text { aleatorio }\end{array}$ & EC & $\begin{array}{l}14 \text { sesiones durante } \\
7 \quad \text { semanas } \quad \text { (dos } \\
\text { semanales), siguiendo } \\
\text { lineamiento de Spector et } \\
\text { al. (2005). }\end{array}$ & $\begin{array}{l}\text { Hay mejoría significativa en recuerdo } \\
\text { libre a largo plazo, memoria visual, } \\
\text { orientación y comprensión auditiva. } \\
\text { Hay dos hipótesis explicativas: la } \\
\text { naturaleza lingüística de la EC mejora } \\
\text { los circuitos asociados a la sintaxis que } \\
\text { puede favorecer los resultados, y la } \\
\text { desestigmatización generada por la EC, } \\
\text { impacta en la memoria y el lenguaje. }\end{array}$ \\
\hline $\begin{array}{l}\text { Spector, A., } \\
\text { Orrell, M. \& } \\
\text { Woods, B. }\end{array}$ & 2010 & $\begin{array}{l}\text { Ensayo } \\
\text { controlado } \\
\text { aleatorio }\end{array}$ & EC & $\begin{array}{l}14 \quad \text { sesiones durante } \\
7 \quad \text { semanas } \quad \text { (dos } \\
\text { semanales), siguiendo } \\
\text { lineamiento de Spector et } \\
\text { al. (2005). }\end{array}$ & $\begin{array}{l}\text { A partir de la EC, se observaron } \\
\text { mejoría en cognición y calidad de vida, } \\
\text { independiente de si el paciente seguía } \\
\text { tratamiento con AChE o no. Estos } \\
\text { efectos se vieron asociados con la edad } \\
\text { avanzada y el sexo femenino. }\end{array}$ \\
\hline $\begin{array}{l}\text { Fernández-Calvo, } \\
\text { B., Contador, } \\
\text { I., Serna, A., } \\
\text { Menezes de } \\
\text { Lucena, V. \& } \\
\text { Ramos, F. }\end{array}$ & 2010 & $\begin{array}{l}\text { Ensayo } \\
\text { controlado } \\
\text { aleatorio }\end{array}$ & $\begin{array}{l}\text { EC y } \\
\text { entrenamiento } \\
\text { a cuidadores }\end{array}$ & $\begin{array}{l}\text { Dos sesiones semanales } \\
\text { durante } 7 \text { semanas } \\
\text { trabajando actividades } \\
\text { listadas por Spector } \\
\text { et al. (2005), y con } \\
\text { el entrenamiento a } \\
\text { cuidadores siguiendo } \\
\text { el manual de Aguirre, } \\
\text { Spector, Streater, Hoe, } \\
\text { Woods y Orrell (2011). }\end{array}$ & $\begin{array}{l}\text { No se obtuvieron cambios significativos } \\
\text { en medidas de cognición, calidad de } \\
\text { vida y calidad de relación entre paciente } \\
\text { y cuidador. Es posible que medidas } \\
\text { semanales de EC no sean suficientes } \\
\text { para generar resultados esperados. }\end{array}$ \\
\hline $\begin{array}{l}\text { Spector, A., } \\
\text { Woods, B. \& } \\
\text { Orrell, M. }\end{array}$ & 2008 & $\begin{array}{l}\text { Ensayo } \\
\text { controlado } \\
\text { aleatorio }\end{array}$ & $\begin{array}{l}\text { Terapia de } \\
\text { mantenimiento } \\
\text { de EC }\end{array}$ & $\begin{array}{l}24 \text { semanas siguiendo } \\
\text { parámetros de la terapia } \\
\text { por EC de Spector et al. } \\
(2003)\end{array}$ & $\begin{array}{l}\text { Se encontraron beneficios en } \\
\text { funcionamiento cognitivo y calidad de } \\
\text { vida luego de } 6 \text { meses en terapia de } \\
\text { mantenimiento por EC en pacientes que } \\
\text { llevaban tratamiento farmacológico con } \\
\text { inhibidores de AChE. }\end{array}$ \\
\hline $\begin{array}{l}\text { Basak, C., Boot, } \\
\text { W., Voss, M. \& } \\
\text { Kramer, A. }\end{array}$ & 2008 & $\begin{array}{l}\text { Ensayo } \\
\text { controlado } \\
\text { aleatorio }\end{array}$ & $\begin{array}{l}\text { EC y } \\
\text { tratamiento con } \\
\text { donepezilo }\end{array}$ & $\begin{array}{l}\text { Dos sesiones semanales } \\
\text { de } 45 \text { minutos por } 7 \\
\text { semanas }\end{array}$ & $\begin{array}{l}\text { La terapia por EC es rentable cuando } \\
\text { se observa la calidad de vida luego de } \\
6 \text { meses como resultado principal, y la } \\
\text { mejora en el funcionamiento cognitivo } \\
\text { como resultado secundario. Ambas } \\
\text { demuestran más beneficios que la } \\
\text { intervención usual recibida en centros } \\
\text { comunitarios de salud. }\end{array}$ \\
\hline Matsuda, $\mathrm{O}$. & 2007 & $\begin{array}{l}\text { Ensayo } \\
\text { controlado } \\
\text { aleatorio }\end{array}$ & EC & $\begin{array}{l}\text { I n t e r v e n c i o n e s } \\
\text { semanales durante } 8 \text { y } 10 \\
\text { semanas consecutivas. }\end{array}$ & $\begin{array}{l}\text { Los síntomas de depresión y agitación } \\
\text { se beneficiaron significativamente de la } \\
\text { aromaterapia por encima de la EC y TR. }\end{array}$ \\
\hline $\begin{array}{l}\text { Requena, C., } \\
\text { Maestú, F., } \\
\text { Campo, P., } \\
\text { Fernández, A. } \\
\text { Gil, P. \& Ortiz, T. }\end{array}$ & 2007 & $\begin{array}{c}\text { Ensayo } \\
\text { controlado } \\
\text { aleatorio }\end{array}$ & $\begin{array}{l}\text { Estimulación } \\
\text { sensorial y } \\
\text { multisensorial, } \\
\text { terapia } \\
\text { conductual, } \\
\text { terapia asistida } \\
\text { por animales, } \\
\text { terapia de } \\
\text { adaptación } \\
\text { al hogar, TR, } \\
\text { TOR, Terapia de } \\
\text { validación }\end{array}$ & Ninguno & $\begin{array}{l}\text { El personal de cuidado y las familias } \\
\text { son parte integral de las estrategias } \\
\text { de tratamiento y son esenciales para } \\
\text { obtener información confiable y construir } \\
\text { intervenciones apropiadas. }\end{array}$ \\
\hline
\end{tabular}


Revista Búsqueda - Julio / Diciembre de 2017

\begin{tabular}{|c|c|c|c|c|c|}
\hline Autores & Año & Método & Terapia & Detalles de la terapia & Hallazgos principales \\
\hline $\begin{array}{l}\text { Tárraga, L., } \\
\text { Boada, M., } \\
\text { Modinos, G., } \\
\text { Espinosa, A., } \\
\text { Diego, S., et. Al }\end{array}$ & 2006 & $\begin{array}{l}\text { Ensayo } \\
\text { controlado } \\
\text { aleatorio }\end{array}$ & EC & $\begin{array}{l}\text { Sesiones grupales de } \\
45 \text { minutos, dos días } \\
\text { a la semana durante } 7 \\
\text { semanas. Se trabajaban } \\
\text { actividades listadas por } \\
\text { Spector et al. (2005). }\end{array}$ & $\begin{array}{l}\text { Se obtienen cambios significativos en } \\
\text { las puntuaciones del SLUMS y de la } \\
\text { Escala de Cornell para la Depresión } \\
\text { en la demencia, sin observar cambios } \\
\text { significativos en las puntuaciones de la } \\
\text { calidad de vida. }\end{array}$ \\
\hline $\begin{array}{l}\text { Metzler- } \\
\text { Baddeley, C. \& } \\
\text { Snowden, J. S. }\end{array}$ & 2005 & $\begin{array}{l}\text { Ensayo } \\
\text { controlado } \\
\text { aleatorio }\end{array}$ & EC individual & $\begin{array}{l}75 \text { sesiones de } 30 \\
\text { minutos durante } 25 \\
\text { semanas en modalidad } \\
\text { individual aplicada por } \\
\text { cuidador en casa, o } \\
\text { en tratamiento usual, } \\
\text { en ámbito clínico por } \\
\text { profesional. Se aplicaron } \\
\text { actividades temáticas. }\end{array}$ & $\begin{array}{l}\text { La EC individual liderada por cuidadores } \\
\text { arrojó cambios favorables en el } \\
\text { funcionamiento cognitivo, pero no en } \\
\text { la calidad de vida de los pacientes. } \\
\text { Empero, auto reportes indican mejoría } \\
\text { de la calidad de vida del cuidador al } \\
\text { liderar la intervención. }\end{array}$ \\
\hline $\begin{array}{l}\text { Loewenstein, D. } \\
\text { A., Acevedo, A., } \\
\text { Czaja, S. J. \& } \\
\text { Duara, R. }\end{array}$ & 2004 & $\begin{array}{l}\text { Ensayo } \\
\text { controlado } \\
\text { aleatorio }\end{array}$ & EC & $\begin{array}{l}\text { Dos sesiones } \\
\text { durante } 7 \quad \text { semanales } \\
\text { trabajando actividades } \\
\text { listadas por Spector et al. } \\
\text { (2005). }\end{array}$ & $\begin{array}{l}\text { La EC es eficaz para el funcionamiento } \\
\text { cognitivo de los pacientes en contexto } \\
\text { italiano con demencia. También com- } \\
\text { prueban aumento de calidad de vida de } \\
\text { los pacientes en el grupo experimental. }\end{array}$ \\
\hline $\begin{array}{l}\text { Spector, A., } \\
\text { Thorgrimsen, } \\
\text { L., Woods, } \\
\text { B., Royan, L., } \\
\text { Davies, S., } \\
\text { Butterworth, M. } \\
\text { \& Orrel, M. }\end{array}$ & 2003 & $\begin{array}{l}\text { Ensayo } \\
\text { controlado } \\
\text { aleatorio }\end{array}$ & Musicoterapia & $\begin{array}{l}2 \text { sesiones semanales de } \\
45 \text { minutos }\end{array}$ & $\begin{array}{l}\text { La musicoterapia mejoró algunas } \\
\text { alteraciones cognitivas, psicológicas } \\
\text { y conductuales de los pacientes con } \\
\text { enfermedad de Alzheimer. }\end{array}$ \\
\hline Arkin, S. M. & 2001 & $\begin{array}{l}\text { Ensayo } \\
\text { controlado no } \\
\text { aleatorio }\end{array}$ & $\begin{array}{l}\text { TR, Terapia de } \\
\text { Validación }\end{array}$ & $\begin{array}{l}1 \text { a } 2 \text { veces semanales } \\
\text { de } 30 \text { a } 90 \text { minutos }\end{array}$ & $\begin{array}{l}\text { Las terapias cognitivas pueden mejorar } \\
\text { el rendimiento mental en la enfermedad } \\
\text { de Alzheimer leve y moderada, ayudando } \\
\text { a la orientación de espacio y tiempo. }\end{array}$ \\
\hline
\end{tabular}

Fuente: Elaboración propia

\section{Revisión de la eficacia de la EC en el TNM y el TNM por EA}

De los tipos de estudios consultados, los de corte experimental (ensayos controlados aleatorios) fueron los más predominantes $(66,2 \%)$. De los estudios revisados, el $98 \%$ obtuvo resultados significativos sobre el efecto de la EC en el funcionamiento cognitivo de la población objetivo, y solo un estudio encontró que no había una mejoría significativa en las puntuaciones de los instrumentos aplicados. Los estudios experimentales estudiaban el efecto de la EC en el funcionamiento cognitivo y en otros aspectos como la calidad de vida, el estado de ánimo, los síntomas neuropsiquiátricos y la independencia. Del $48 \%$ de los estudios que investigaron acerca del efecto de la EC en la calidad de vida, el $70 \%$ de estos obtuvo resultados significativos. En cuanto al estado de ánimo, del $19 \%$ de investigaciones que estudiaron esta área, el $50 \%$ de estudios obtuvo resultados significativos. Del $24 \%$ de las investigaciones que estudiaron los efectos de la EC en los síntomas neuropsiquiátricos de la EA y del TNM por $E A$, el $60 \%$ obtuvo resultados significativos. Por último, solo el $9 \%$ estudió el efecto de la terapia en la independencia, sin embargo, ninguna de estas investigaciones encontró resultados significativos. 
En cuanto a la extensión de las intervenciones por EC, se observó una heterogeneidad en los datos, pues los programas de EC varían entre siete y veinticinco semanas, mientras hay un $12 \%$ de estudios que no especifican este aspecto en su metodología. El $50 \%$ de las investigaciones revisadas emplea intervenciones entre siete y doce semanas, pues implementan protocolos de EC de Spector et al. (2005). Esta información demuestra que hay un alto porcentaje de estudios que implementan intervenciones por diseño propio. El $48 \%$ de los estudios implementan alguno de estos dos, mientras que el $45 \%$ diseña su propio formato y el $7 \%$ no establece con exactitud este hecho.

En cuanto a las tareas implementadas en los programas de EC, solo el $27 \%$ de las investigaciones detalla qué tipo de actividades se trabajan y qué dominio cognitivo se pretende estimular con dicha propuesta. De este $27 \%$, el $31 \%$ de los estudios tiene como objetivo estimular la memoria, el $30 \%$ busca trabajar el dominio de lenguaje, el $17 \%$ pretende fortalecer procesos de función ejecutiva, el $18 \%$ implementa tareas para trabajar praxias y atención, y el $4 \%$ restante, lo hace con percepción.

Como se puede apreciar en los resultados, varios autores evalúan la eficacia de la EC en conjunto con la eficacia de tratamientos farmacológicos con inhibidores de acetilcolina. De la muestra de estudios consultados, el $41 \%$ indagó acerca de esto, y de ese porcentaje de investigaciones declaradas, el $55 \%$ de estos estudios encontró que la EC trae beneficios mayores a los tratamientos farmacológicos.

\section{Discusión}

La Terapia Rememorativa es un tipo de intervención no farmacológica que busca generar espacios de reflexión al rememorar eventos del pasado, tanto positivos como tensionantes, para dotar de nuevos significados a esos recuerdos y realizar una conversión terapéutica de experiencias vividas hacia un proceso de adaptación y resiliencia (GonzálezArévalo, 2015).

En Colombia, las cifras de prevalencia del TNM oscilan entre el 1,8\% y 3,4\% en personas entre los 65 y 75 años (Ruiz de Sánchez et al., 2010). A pesar de la importancia de estudiar y tratar esta población al constituirse como una problemática de salud pública, no se encontraron estudios epidemiológicos sobre el TNM por EA en Colombia, aun cuando se conoce que la EA es la principal causa de TNM a nivel mundial (Huff, 2016).

Esta enfermedad se caracteriza por producir una degeneración celular a nivel central como proceso secundario de la amiloidogénesis y la degeneración neurofibrilar, que son específicos de la EA (Huff, 2016). Por estos dos procesos hay alteraciones cognitivas, comportamentales y pérdida de independencia (Asociación Americana de Psiquiatría, 2014) que incapacitan al paciente y traen consecuencias para su familia y la sociedad (Cicerone et al., 2000; Shankar, 2008; Hart, 2010).

En la actualidad es difícil encontrar tratamientos definitivos que curen el proceso neurodegenerativo de la EA ni del TNM por EA (Requena et al., 2007). No obstante, hay tratamientos farmacológicos y no farmacológicos que se utilizan para disminuir la velocidad de la progresión de los cuadros de TNM (Buschert et al., 2010; Takeda, Tanaka, Okochi y Kazu, 2012; Woods et al., 2012). La EC encabeza las terapias cognitivas de preferencia (Ruiz-Sánchez de León, 2012), y la aplican los neuropsicólogos dentro de contextos de intervención 
terapéutica (Ballard, Khan, Clack y Corbett, 2011; Saykin y Rabin, 2016), lo que le ha hecho tener un papel importante en el trabajo clínico e investigativo con los pacientes diagnosticados con TNM por EA.

La EC es una terapia no farmacológica empleada en la intervención de TNM por EA en estadios leves y moderados para fortalecer el funcionamiento de los dominios cognitivos asociados a esta patología (Tárraga et al., 2006; Fernández-Calvo et al., 2010;). Es rentable (Spector, Woods y Orrell, 2008; Fernández-Calvo et al., 2010; Olazarán et al., 2010; D'Amico et al., 2015) y beneficia tanto la actividad cognitiva como la calidad de vida, las habilidades comunicativas y la interacción social de las personas diagnosticadas con esta condición (Spector et al., 2003; Woods et al., 2012), cuando se trabajan de manera global todos los dominios cognitivos (Buschert et al., 2010).

Los resultados de la revisión demostraron que solo el $48 \%$ de los estudios se basan en protocolos de intervención para delimitar su programa de intervención, exponiendo como principal limitación el acceso restringido a protocolos que guíen la implementación de esta terapia bajo lineamientos basados en la evidencia (Khan et al., 2014). Por otro lado, no se encontraron protocolos colombianos de EC para pacientes con EA y TNM por EA.

Los resultados exponen que el $98 \%$ de las investigaciones consultadas demostró eficacia de la terapia por EC a corto y a largo plazo (desde siete hasta veinticinco meses). Muchos estudios aplicaron los protocolos de los manuales de Spector et al. (2005) y Aguirre et al. (2011) para implementar terapias por EC y terapias de mantenimiento por EC, mientras otros desarrollaron programas de intervención utilizando otras tareas, como juegos disponibles en el mercado, y actividades que conceptual y teóricamente cumplen con la definición de la EC. Así, se evidencia una heterogeneidad amplia en las metodologías implementadas: en cuanto a extensión de la intervención, la duración de las sesiones y las actividades realizadas en el programa.

Al comparar medidas pre y post test, luego de un programa de EC, se observan efectos positivos en el funcionamiento cognitivo de los pacientes diagnosticados con TNM por EA en estadios leves o moderados (Tárraga et al., 2006; Requena et al., 2007), con obtención de resultados favorables e incluso superiores a los de los tratamientos farmacológicos con inhibidores de AChE en $55 \%$ de los estudios que lo investigaron (Matsuda et al., 2010; Kurz et al., 2011; Woods et al., 2012; Aguirre et al., 2013).

Por otro lado, se observaron varias limitaciones en la revisión que comprometen la demostración de la eficacia de la EC en el TNM por EA con un volumen amplio de estudios que lo comprueben. Entre estas limitaciones se encuentran: la ausencia de investigaciones que valoren la eficacia de las actividades particulares que se aplican en intervenciones por EC -considerando que la mayoría de estudios emplean intervenciones por diseño propio- $y$ las afirmaciones de varios autores que manifiestas condiciones estadísticas y metodológica que afectan la calidad de los resultados de la mayoría de los estudios del campo (tamaño de muestra, instrumentos no validados empleados, calidad de la muestra) (Kurz et al., 2011; Woods et al., 2012; D'onofrio etal., 2016). En lo que respecta a esta revisión, cabe anotar que se utilizaron únicamente dos bases de datos. Esto puede representar un problema ya que el aumento de bases de datos podría haber aumentado la cantidad de artículos revisados. Sin 
embargo, las dos bases que se utilizaron tienen la particularidad de recoger la mayoría de las investigaciones dedicadas al estudio de la estimulación cognitiva en los trastornos cognitivos mayores.

Las limitaciones anteriores, en conjunto con la necesidad imperante de estudiar el TNM por EA en Colombia, establecen que este es un campo que debe explorarse a profundidad con urgencia, especialmente en cuanto a la intervención de esta población. Se pretende implantar la necesidad en otros profesionales por animarse a contribuir con el campo de estudio, y con las personas que requieren la asistencia hoy y a futuro.

\section{Referencias bibliográficas}

Aguirre, E.; Hoare, Z.; Streater, A.; Spector, A.; Woods, B.; Hoe, J. y Orrell, M. (2013). Cognitive stimulation therapy (CST) for people with dementia. Who benefits most? International Journal of Geriatric Psychiatry, 28(3), 284-290. doi: 10.1002/gps.3823.

Alzheimer's Association. (2017). Alzheimer's disease facts and figures. Alzheimer's y Dementia, 13(4), 325-373.

Arkin, S.M. (2001). Alzheimer rehabilitation by students: interventions and outcomes. Neuropsychological Rehabilitation, 11(3/4), 273-317. doi: 10.1080/09602010143000059.

Asociación Americana de Psiquiatría. (2014). Manual Diagnóstico y Estadístico de los Trastornos Mentales (DSM-5®). Madrid: Editorial Médica Panamericana.

Asociación de Alzheimer (2010). Reporte Especial: Raza, Etnicidad y la Enfermedad de Alzheimer: Extracto de "Hechos y Cifras de la Enfermedad de Alzheimer 2010 (2010 Alzheimer's Disease Facts and Figures') de la Asociación de Alzheimer. Recuperado de: https:// www.alz.org/documents_custom/ ALZ_FF2010_Spanish_03.09.10.pdf
Bäckman, L.; Jones, S.; Berger, A.; Jonsson, E. y Small, B.J. (2005). Cognitive impairment in preclinical Alzheimer's Disease: A MetaAnalysis. Neuropsychology, 19(4), 520-531. Doi: 10.1037/08944105.19.4.520.

Ballard, C.; Khan, Z.; Clack, H. y Corbett, A. (2011). Nonpharmacological treatment of Alzheimer's Disease. The Canadian Journal of Psychiatry, 56(10), 589595. doi: https:// doi.org/10.11 77/070674371105601004.

Basak, C.; Boot, W.; Voss, M. y Kramer, A., (2008). Can training in a realtime strategy videogame attenuate cognitive decline in older adults? Psychological Aging, 23(4), 765777. Doi:10.1037/a0013494.

Benveniste, S.; Jouvelot, P.; Pin, B. y Péquignot, R. (2012). The MINWii project: renarcissization of patients suffering from Alzheimer's disease through video game-based music therapy. Entertainment Computing, 3,111-120. doi: 10.1016/j. entcom.2011.12.004.

Buschert, V.; Bokde, A.L.W. y Hampel, H. (2010). Cognitive intervention in Alzheimer's Disease. Nature Reviews Neurology, 6(9), 508-517. doi: 10.1038/nrneurol.2010.113. 
Capotosto, E.; Belacchi, C.; Gardini, S.; Faggian, S.; Piras, F.; Mantoan V.; Salvalaio, E.; Pradelli, S. y Borella, E. (2017). Cognitive stimulation therapy in the Italian context: its efficacy in cognitive and noncognitive measures in older adults with dementia. International Journal of Geriatric Psychiatry, 32(3):331340. doi: $10.1002 / g p s .4521$

Chodos, A.; Johnston, B. y Yaffe, K. (2014). Dementia and Delirium. En M.D. Feldman, J.F. Christensen y J.M. Satterfield (eds.). Behavioral Medicine: a Guide for Clinical Practice. Nueva York: McGraw-Hill.

Cicerone, K.D.; Dahlberg, C.; Kalmar, K.; Langenbahn, D.M.; Malec, J.F.; Bergquist, T.F.; Felicetti, T.; Giacino, J.T.; Harley, J.P.; Harrington, D.E.; Herzog, J.; Kneipp, S.; Laatsch, L. y Morse, P.A. (2000). Evidencebased cognitive rehabilitation: recommendations for clinical practice. Archives for Physical Medical Rehabilitation, 81(12), 1596-1615. doi: 10.1053/apmr.2000.19240.

Clare, L.; Wilson, B. A.; Carter, G.; Roth, I. y Hodges, J.R. (2004). Awareness in early stage Alzheimer's Disease: relationship to outcome cognitive rehabilitation. Journal of Clinical and Experimental Neuropsychology, 26(2), 215-226. doi: http://dx.doi. org/10.1076/jcen.26.2.215.28088.

Cove, J.; Jacobi, N.; Donovan, H.; Orrell, M.; Stott, J. y Spector, A. (2014). Effectiveness of weekly cognitive stimulation therapy for people with dementia and the additional impact of enhancing cognitive stimulation therapy with a career training program. Clinical Interventions in Aging, 9, 2143-2150. doi: http:// dx.doi.org/10.2147/CIA.S66232.
D'Amico, F.; Rehill, A.; Knapp, M.; Aguirre, E.; Donovan, H.; Hoare, Z.; Hoe, J.; Russell, I.; Spector, A.; Streater, A.; Whitaker, C.; Woods, R.T. y Orrell, M. (2015). Maintenance Cognitive Stimulation Therapy: an Economic Evaluation Within a Randomized Controlled Trial. Journal of the American Medical Directors Association, 16(1), 6370. doi: https://doi.org/10.1016/j. jamda.2014.10.020.

D'Onofrio, G.; Sancarlo, D.; Seripa, D.; Ricciardi, F.; Giuliani, F.; Panza, F. y Greco, A. (2016). NonPharmacological Approaches in the Treatment of Dementia. En D. Vito (ed.), Update on Dementia. doi: $10.5772 / 64232$.

De Sánchez, C. R., Nariño, D., \& Cerón, J. F. M. (2010). Epidemiología y carga de la Enfermedad de Alzheimer. Acta Neurológica Colombiana, 26(3), 1.

Fernández-Calvo, B.; Contador, I.; Serna, A; Menezes de Lucena, V. y Ramos, F. (2010). El efecto del formato de intervención individual o grupal en la estimulación cognitiva de pacientes con Enfermedad de Alzheimer. Revista de Psicopatología y Psicología Clínica, 15(2), 115-123.

Gómez. M. y Gómez, J. (2017). Musicoterapia en la enfermedad de Alzheimer: efectos cognitivos, psicológicos y conductuales. Sociedad española de neurología, 32(5), 300-308. doi: doi.org/10.1016/j. nrl.2015.12.003.

González-Arévalo, K.A. (2015). Terapia de reminiscencia y sus efectos en los pacientes mayores con demencia. Psicogeriatría, 5(3), 101-111.

Guétin, S. ; Portet, F. ; Picot, M.C.; Pommié, C.; Messaoudi, M.; Djabelkir, L.; 
Orejarena-Ballestas et al. Estimulación para pacientes con trastorno por enfermedad de Alzheimer

Olsen, A.L.; Cano, M.M.; Lecourt, E. y Touchon, J. (2009). Effect of Music Therapy on Anxiety and Depression in Patients with Alzheimer's Type Dementia: Randomized, Controlled Study. Dementia and Geriatric Cognitive Disorders, 28, 36-46. Doi: 10.1159/000229024.

Hall, L.; Orrell, M.; Stott, J. y Spector, A. (2013). Cognitive stimulation therapy (CST): neuropsychological mechanisms of change. International Psychogeriatrics, 25(3), 479-489. doi: $10.1017 /$ S1041610212001822.

Hart, T. (2010). Cognitive rehabilitation. En R.G. Frank, M. Rosenthal y B. Caplan (eds.). Handbook of Rehabilitation Psychology. American Psychological Association (pp. 285-301).

Kane, R.L. (2013). Chapter 6. Delirium and Dementias. En R.L. Kane, J.G. Ouslander, I.B. Abrass y B. Resnick (eds.). Essentials of Clinical Geriatrics. Nueva York: McGraw-Hill.

Khan, Z., Corbett, A. y Ballard, C. (2014). Terapia de estimulación cognitiva: entrenamiento, mantenimiento e implementación en ensayos clínicos. Pragmatic and Observational Research, 5, 15-19. doi: https://doi. org/10.2147/POR.S56000.

Kirova, A.; Bays, R.B. y Lagalwar, S. (2015). Working memory and executive function decline across normal aging, Mild Cognitive Impairment and Alzheimer's Disease. BioMed Research International, 1-9. doi: $10.1155 / 2015 / 748212$.

Kurz, A.F.; Leucht, S. y Lautenschlager, N.T. (2011). The clinical significance of cognition-focused interventions for cognitively impaired older adults: a systematic review of randomized controlled trials. International
Psychogeriatrics, 23(9), 1364-1375. doi: $10.1017 / S 1041610211001001$.

Loewenstein, D.A.; Acevedo, A.; Czaja, S.J. y Duara, R. (2004). Cognitive rehabilitation of mildly impaired Alzheimer Disease patients on Cholinesterase inhibitors. American Journal of Geriatric Psychiatry, 12(4), 395-402. doi: 10.1176/appi. ajgp.12.4.395.

Luján, I. (2016, enero 12). La fisiopatología del Alzheimer: últimos avances. Universitat de Valencia. Recuperado de: http:// www.uv.es/uvweb/masterfisiologia/es/blog/fisiopatologiadel-alzheimer-ultimos-avances1285952573044/GasetaRecerca. html?id=1285954369657

Maci, T.; Le Pira, F.; Quattrocchi, G.; Di Nuovo, S.; Perciavalle, V. y Zappia, M. (2012). Physical and Cognitive Stimulation in Alzheimer Disease. The GAIA Project: A Pilot Study. American Journal of Alzheimer's Disease $y$ Other Dementias, 27(2), 107-113. doi: $10.1177 / 1533317512440493$.

Matsuda, O. (2007). Cognitive stimulation therapy for Alzheimer's disease: the effect of cognitive stimulation therapy on the progression of mild Alzheimer's disease in patients treated with donepezil. International Psychogeriatrics, 19(2), 241-252. doi: https://doi.org/10.1017/ S1041610206004194.

Matsuda, O.; Shido, E.; Hashikai, A.; Shibuya, H.; Kuono, M.; Hara, C. y Saito, M. (2010). Short-term effect of combined drug therapy and cognitive stimulation therapy on the cognitive function of Alzheimer's Disease. Psychogeriatrics, 10, 167-172. doi: 10.1111/j.14798301.2010.00335.x. 
Mesulam, M. (2015). Aphasia, Memory Loss, and Other Focal Cerebral Disorders. En D.L. Kasper, A.S. Fauci, S.L. Hauser, D.L. Longo, J.L. Jameson y J. Loscalzo (eds.). Harrison's principles of Internal Medicine. Nueva York: McGrawHill. Recuperado de: http:// accessmedicine.mhmedical.com.ez. unisabana.edu.co/ content.aspx?b ookid $=1130$ ysectionid $=79724975$.

Metzler-Baddeley, C. y Snowden, J.S. (2005). Brief report: Errorless vs. Errorful learning as a memory rehabilitation approach in Alzheimer's Disease. Journal of Clinical and Experimental Neuropsychology, 27, 1079-1079. doi: $10.1080 / 13803390490919164$.

National Centre for Biotechnology Information. (2017). Non-drug interventions for Alzheimer's Disease. Recuperado de: https://www. ncbi.nlm.nih.gov/pubmedhealth/ PMH0072539/\#i2219. behandlungpy.cognitivetherapyappr

Niu, Y.; Tan, J.; Guan, J.; Zhang, Z. y Wang, L. (2010). Cognitive stimulation therapy in the treatment of neuropsychiatric symptoms in Alzheimer's disease: a randomized controlled trial. Clinical Rehabilitation, 24(12), 1101-1111. doi: $10.1177 / 0269215510376004$.

Olazarán, J.; Reisberg, B.; Clare, L.; Cruz, I.; Peña-Casanova, J.; Del Ser, T.; Woods, B.; Beck, C.; Auer, S.; Lai, C.; Spector, A.; Fazio, S.; Bond, J.; Kivipelto, M.; Brodaty, J.; Rojo, J.M.; Collins, H.; Teri, L.; Mittelman, M.; Orrell, M.; Feldman, H.H. y Muñiz, R. (2010). Nonpharmacological therapies in Alzheimer's Disease: a systematic review of efficacy. Dementia and Geriatric Cognitive
Disorders, 30, 161-178. doi: https:// doi.org/10.1159/000316119.

Orrell, M.; Aguirre, E.; Spector, A.; Hoare, Z.; Woods, R.T.; Streater, A.; Donovan, H.; Hoe, J., Knapp, M.; Whitaker, C. y Russell, I. (2014). British Journal of Psychiatry, 204(6), 454-461. doi: 10.1192/bjp. bp.113.137414.

Requena, C.; Maestú, F.; Campo, P.; Fernández, A.; Gil, P. y Ortiz, T. (2007). Efectos del tratamiento combinado de fármaco más estimulación cognitiva en la demencia moderada: seguimiento de dos años. Revista Española de Geriatría y Gerontología, 42(1), 3-10- doi: https://doi.org/10.1016/ S0211-139X(07)73515-1.

Romero, E. (2002). Orientación, memoria y aprendizaje. En E. Romero y G. Vázquez (eds.) Actualización en Psicología Clínica. Buenos Aires: Ediciones GeKa (pp. 85-105).

Ruíz de Sánchez, C. R., Nariño, D., \& Muñoz, J. F. (2010). Epidemiología y carga de la Enfermedad de Alzheimer. Acta Neurol Colomb, 26(3), 1.

Ruiz-Sánchez de León, J.M. (2012). Estimulación cognitiva en el envejecimiento sano, el deterioro cognitivo leve y las demencias: estrategias de intervención y consideraciones teóricas para la práctica clínica. Revista de Logopenia, Foniatría y Audiología, 32, 57-66. doi: $10.1016 /$ j.rlfa.2012.02.002.

Särkämö, T. (2017). Cognitive, emotional and neural benefits of musical leisure activities in aging and neurological rehabilitation: A critical review. Annals of Physical and Rehabilitation Medicine. doi: http://dx.doi. org/10.1016/j.rehab.2017.03.006. 
Saykin, A.J.yRabin,L.A. (2014). Dementias and neurodegenerative diseases. En M.W. Parsons y T.A. Hammeke (eds.). Clinical neuropsychology: a pocket handbook for assessment. American Psychological Association (pp.237-266).

Shankar, G.M.; Li, S.; Mehta, T.H.; García-Muñoz, A.; Shepardson, N.E.; Smith, I.; Brett, F.M.; Farrell, M.A.; Rowan, M.J.; Lemere, C.A.; Regan, C.M.; Walsh, D.M.; Sabatini, B.L. y Selkoe, D.J. (2008). Amyloid $\beta$-Protein dimers isolated directly from Alzheimer's brains impair synaptic plasticity and memory. Nature Medicine, 14(8), 837-842. doi: $10.1038 / \mathrm{nm} 1782$.

Spector, A.; Hoe, J.; Russell, I.T.; Knapp, M.; Woods, R.T. y Orrell, M. (2010). Maintenance Cognitive Stimulation Therapy (CST) for dementia: A singleblind, multi-centre, randomized controlled trial of Maintenance CST vs. CST for dementia. Trial, 11(46), 1-10. doi: https://doi. org/10.1186/1745-6215-11-46.

Spector, A.; Streater, A.; Hoe, J.; Woods, B. y Orrell, M. (2011). Making a Difference 2. Hawker Publications: UK.

Spector, A.; Thorgrimsen, L.; Woods, B.; Royan, L.; Davies, S.; Butterworth, M. y Orrell, M. (2003). Efficacy of an evidence-based cognitive stimulation therapy programme for people with dementia. British Journal of Psychiatry, 183, 248-254.

Stewart, D.B.; Berg-Weger, M.; Tebb, S.; Sakamoto, M.; Roselle, K.; Downing, L.; Lundy, J. y Hayden, D. (2017). Making a Difference: a Study of Cognitive Stimulation Therapy for Persons with Dementia. Journal of gerontological Social Work, 60(4), 300-312. doi: 10.1080/01634372.2017.1318196.
Takeda, M. ; Tanaka, T.; Okochi, M. y Kazui, H. (2012). Non-pharmacological intervention for dementia patients. PsychiatryandClinical Neurosciences, 66(1), 1-7. doi: 10.1111/j.14401819.2011.02304.x.

Tárraga, L.; Boada, M.; Modinos, G.; Espinosa, A.; Diego, S.; Morera, A.; Guitart, M.; Balcells, J.; López, O.L. y Becker, J.T. (2006). A randomised pilot study to assess the efficacy of an interactive, multimedia tool of cognitive stimulation in Alzheimer's Disease. Journal of Neurology, Neurosurgery and Psychiatry, 77(10), 1116-1121. doi: 10.1136/ jnnp.2005.086074.

Thorgrimsen, L.; Woods, B. y Orrell, M. (2005). Our Time: an EvidenceBased Group Program to Offer Cognitive Stimulation to People with Dementia. Manual for Group Leaders. Cedar Falls: Freiberg Press.

Woods, B. y Orrell, M. (2008). Cognitive stimulation for the treatment of Alzheimer's Disease. Expert Review of Neurotherapeutics, 8(5), 751-757. doi: $10.1586 / 14737175.8 .5 .751$

Woods, B.; Aguirre, E.; Spector, A.E. y Orrell, M. (2012). Cognitive stimulation to improve cognitive functioning in people with dementia [Review]. The Cochrane Library, 2, 1-56.

Woods, R.T.: Spector, A. y Orrell, M. (2013). Cognitive stimulation for dementia: a systematic review of the evidence of effectiveness from randomised controlled trials. Ageing Research Reviews, 12(1), 252-262. doi: https://doi.org/10.1016/j. arr.2012.07.001.

Yamanaka, K.; Kawano, Y.; Noguchi, D.; Nakaaki, S.; Watanabe, N.; Amano, T. y Spector, A. (2013). Effects 
of cognitive stimulation therapy Japanese version (CST-J) for people with dementia: a single-blind, controlled clinical trial. Aging and Mental Health, 17(5), 579-586. doi: 10.1080/13607863.2013.777395.

Yang, Y.P.; Lee, F.P.; Chao, H.C.; Hsu, F.Y. y Wang, J.J. (2016). Comparing the Effects of Cognitive Stimulation, Reminiscence, and Aroma-Massage on Agitation and Depressive Mood in People With Dementia. Journal of the American Medical Directors Association, 17(8), 719-24. doi: 10.1016/j.jamda.2016.03.021.
Yates, L.; Leung, P.; Kang, S.; Hoare, Z.; Whitaker, C.; Burns, A.; Knapp, M.; Leroi, I.; Moniz-Cook, E.; Pearson, S.; Simpson, S.; Spector, A.; Roberts, S.; Russell, I.; De Waal, H.; Woods, R.T. y Orgeta, V. (2017). The impact of individual Cognitive Stimulation Therapy (iCST) on cognition, quality of life, caregiver health, and family relationships in dementia: a randomised controlled trial. PLoS Medicine, 14(3). doi: 10.1371/journal.pmed.1002269. 